\title{
Impact of orange juice consumption on macronutrient and energy intakes and body composition in the US population
}

\author{
Ying Wang ${ }^{1}$, Beate Lloyd ${ }^{2}$, Meng Yang ${ }^{1}$, Catherine G Davis ${ }^{1}$, Sang-Gil Lee ${ }^{1}$, \\ Wutae Lee ${ }^{3}$, Sang-Jin Chung ${ }^{4}$ and Ock K Chun ${ }^{1, *}$ \\ 'Department of Nutritional Sciences, University of Connecticut, 3624 Horsebarn Road Extension Unit 4017, \\ Storrs, CT 06269-4017, USA: ${ }^{2}$ PepsiCo Nutrition, Global Research \& Development, PepsiCo Inc., Valhalla, \\ NY, USA: ${ }^{3}$ Harvard College, Harvard University, Cambridge, MA, USA: ${ }^{4}$ Foods and Nutrition, Kookmin \\ University, Seoul, Republic of Korea
}

Submitted 23 May 2011: Final revision received 31 January 2012: Accepted 7 February 2012: First published online 20 March 2012

\begin{abstract}
Objective: The present study evaluated the contribution of $100 \%$ orange juice (OJ) consumption to the intakes of macronutrients and energy and its impact on body composition.

Design: A cross-sectional study was conducted. The main exposure was OJ consumption based on two non-consecutive $24 \mathrm{~h}$ diet recalls. Macronutrient and energy intakes and body composition parameters were outcome measures. All statistical analyses were carried out using SAS and SUDAAN statistical software packages to allow for multistage sample designs.

Setting: The US population and its subgroups.

Subjects: The US population aged $\geq 4$ years ( $n$ 13971) from the National Health and Nutrition Examination Survey 2003-2006, conducted by the National Center for Health Statistics.

Results: In this US population, OJ consumers had lower BMI and healthier lifestyle behaviours (including lower alcohol consumption and smoking as well as higher exercise level) than non-consumers $(P<0 \cdot 05)$. After adjusting for covariates, OJ consumers had higher daily intakes of carbohydrate, total sugar, total fat and energy than non-consumers $(P<0 \cdot 01)$. However, these linear trends still remained even after OJ was removed from the food list of items consumed. Adult OJ consumers had lower BMI, waist circumference and percentage body fat than non-consumers $(P<0 \cdot 01)$, as well as lower odds ratio for overweight and obesity $(P<0 \cdot 01)$. These effects were not seen in children and adolescents, where there was no significant difference in BMI, waist circumference and percentage body fat in OJ consumers compared with non-consumers.

Conclusions: OJ consumption was associated with healthier body composition in adults; while there were no significant associations between OJ consumption and body composition in children and adolescents.
\end{abstract}

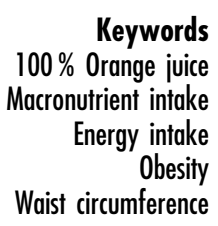

Fruit juice consumption has been increasing over the past several decades for a number of reasons, including an increase in the variety of fruit juice produced and an increase in availability ${ }^{(1)}$. This has prompted concerns from a number of professional groups, including the American Academy of Pediatrics, about fruit juice intake and its potential impact on body weight ${ }^{(2)}$. The scientific data supporting these concerns regarding fruit juice consumption and body weight have been mixed. Several epidemiological studies observed that excessive fruit juice consumption was correlated with an increased risk of obesity in children ${ }^{(1,3-5)}$. In addition, Dennison et al. reported that high intake of apple juice rather than orange juice or other types of $100 \%$ fruit juice was associated with obesity among a group of 2- and 5-year-old children ${ }^{(4)}$. Conversely several other studies have documented no association between fruit juice consumption and overweight or obesity in children ${ }^{(6-10)}$ and have shown that compared with non-consumers, those consuming $100 \%$ fruit juice had overall more nutritious diets including significantly higher intakes of vitamins $\mathrm{C}$ and $\mathrm{B}_{6}, \mathrm{~K}$, riboflavin, $\mathrm{Mg}, \mathrm{Fe}$ and folate and significantly lower intakes of total fat, SFA, discretionary fat and added sugar ${ }^{(8)}$. In addition, children consuming $100 \%$ fruit juice also consumed significantly more servings of total whole fruit than non-consumers ${ }^{(8)}$. These contradictory findings may be partially attributed to some limitations in the scope of previous studies: (i) most of the past studies failed to separate different types of fruit 
juices or separate $100 \%$ fruit juice from juice drinks that contain less than $100 \%$ fruit juice in the evaluation of the impact of juice consumption on body composition ${ }^{(1,5-8,10)}$; (ii) in some studies a single $24 \mathrm{~h}$ diet recall (DR) was used and assumed to reflect usual diet pattern ${ }^{(9,10)}$; (iii) some studies had a small number of participants ${ }^{(6)}$; (iv) some studies used outdated national survey data for the analysis $^{(9,10)}$; and (v) previous studies have not examined the impact of $100 \%$ fruit juice on body composition in adults.

Orange juice is one of the most nutrient-dense fruit juices consumed in the USA and is rich in essential nutrients such as vitamin $\mathrm{C}$, folate, vitamin $\mathrm{B}_{6}$, thiamin, niacin, riboflavin, $\mathrm{K}$ and $\mathrm{Mg}^{(11)}$. In addition, orange juice is a good source of antioxidants including carotenoids and flavonoids such as hesperetin and naringenin ${ }^{(11,12)}$. While orange juice is a nutrient-dense beverage, it has been speculated that it contributes to obesity risk by adding energy and sugars to US diets. However, information on the impact of orange juice on macronutrient and energy intakes, body composition and risk of obesity is limited. Therefore, we aimed to investigate the association of $100 \%$ orange juice consumption with macronutrient and energy intakes and body composition by utilizing a recently released, nationally representative health and nutrition data set of the free-living US population.

\section{Materials and methods}

\section{Data source}

The National Health and Nutrition Examination Survey (NHANES) is conducted by the National Center for Health Statistics to obtain nationally representative information on the health and nutritional status of the US population. NHANES is a national survey involving household interviews and clinical examinations. It was changed in 1999 from a periodic annual survey to a continuous annual survey, and the continuous NHANES data have been released in two-year increments for public use ${ }^{(13)}$.

\section{Participants}

The NHANES uses a stratified, multistage probability sample design and weighting methodology that allows for unbiased national estimates to be produced for the civilian, non-institutionalized US population. The NHANES sample weights adjust for unequal probabilities of selection, nonresponse and planned oversampling of young children, the elderly, low-income persons and ethnic minorities ${ }^{(13)}$.

All interviewed persons were invited to the mobile examination centre, where the $24 \mathrm{~h}$ DR and questionnaires on dietary and lifestyle behaviours were administered. Individuals aged $\geq 4$ years in NHANES 2003-2004(14) and 2005-2006 ${ }^{(15)}$ with reliable and complete DR data were included in the present study ( $n$ 13971). Participants were grouped into subgroups by sociodemographic and lifestyle variables: age (4-8, 9-13, 14-18, 19-30, 31-50,
$>50$ years); gender; ethnicity (non-Hispanic whites, nonHispanic blacks, Mexican Americans, others); BMI $(<18 \cdot 5$, $\left.18 \cdot 5-25 \cdot 0,25 \cdot 0-30 \cdot 0, \geq 30 \cdot 0 \mathrm{~kg} / \mathrm{m}^{2}\right) ;$ poverty income ratio (PIR; <1.3, 1.3-3.5, >3.5); alcohol consumption (yes or no to 'at least 12 alcoholic drinks/year'); current smoking (yes or no to 'current cigarette smoking'); and exercise levels (0, T1, T2, T3; expressed as the MET (metabolic equivalent of task) score calculated by combining the intensity level of the leisure-time activities reported with their mean duration and frequency).

\section{Dietary intake data}

The dietary intake data were estimated from two $24 \mathrm{~h} \mathrm{DR}$ interviews conducted in the NHANES 2003-2004 ${ }^{(14)}$ and 2005-2006 ${ }^{(15)}$, which were carried out by trained interviewers using the US Department of Agriculture (USDA) Automated Multiple Pass Method ${ }^{(16)}$. The day 1 recalls were conducted in person in the NHANES mobile examination centre. The day 2 recalls were conducted by telephone interview approximately 3 to $10 \mathrm{~d}$ after the day 1 recall. Food consumption data were coded using the USDA Food and Nutrient Database for Dietary Studies version 3.0 (FNDDS 3.0) to produce energy and macronutrient intake data. Since a food composition table for added sugar is not available in FNDDS $3 \cdot 0$, a special added sugar database was utilized for estimating added sugar intake ${ }^{(17)}$. In the present study, $100 \%$ orange juice (OJ) included non-sweetened $100 \%$ orange juice and non-sweetened $100 \%$ orange juice fortified with $\mathrm{Ca}$ and vitamin D. OJ consumers were then defined as those who reported they consumed $\mathrm{OJ}$ as a beverage at least once in the two non-consecutive $24 \mathrm{~h}$ DR, while non-consumers were defined as the rest of the participants. OJ consumption was the mean of the two days.

\section{Body composition parameters}

Height, weight and waist circumference (WC) were measured by trained technicians using standardized protocols and calibrated equipment. Fat mass was determined by dual-energy X-ray absorptiometry from whole-body scans administered in the NHANES mobile examination centre. Percentage body fat (body fat \%) was calculated. BMI was calculated and rounded to the nearest $0 \cdot 1 \mathrm{~kg} / \mathrm{m}^{2}$. For adults, BMI categories were defined using widely accepted cut-off points, i.e. $<18.5 \mathrm{~kg} / \mathrm{m}^{2}$ for underweight, $18.5-24.9 \mathrm{~kg} / \mathrm{m}^{2}$ for normal weight, $25 \cdot 0-29 \cdot 9 \mathrm{~kg} / \mathrm{m}^{2}$ for overweight and $\geq 30.0 \mathrm{~kg} / \mathrm{m}^{2}$ for obesity ${ }^{(18)}$. As for the definition of obesity in the US children, the Centers for Disease Control and Prevention (CDC) defined that children with BMI-for-age between the 85th and 95th percentiles are overweight and those with BMI-for-age at or above 95th percentile are obese using the databases that were developed in $1997^{(19)}$. The present study used the same cut-off points for determining overweight and obesity and BMI-for-age $Z$-scores were calculated based on the 2000 CDC BMI-for-age growth charts for US children ${ }^{(20)}$. 


\section{Statistical analyses}

All data analyses was carried out using the SAS statistical software package release 9.2 (2009; SAS Institute Inc., Cary, NC, USA) and the Survey Data Analysis for multistage sample designs professional software package (SUDAAN), release $8 \cdot 0 \cdot 2$ (2003; Research Triangle Institute, Research Triangle Park, NC, USA). Sample weighting was applied to all analyses to account for the unequal probabilities of selection, non-coverage and non-response bias resulting from oversampling of low-income persons, adolescents, the elderly, African Americans and Mexican Americans. SUDDAN was used to increase the reliability and validity of the results through computing variance estimates and test statistics for a stratified, multistage probability survey design.

The $\chi^{2}$ test was applied for assessing the distributions of categorical variables. ANOVA was used to compare means for interval scale variables and to test overall differences in percentages of $\mathrm{OJ}$ consumption by sociodemographic and lifestyle variables. The analysis was stratified by age to examine the association of macronutrient intakes and body composition with OJ consumption. Arithmetic means and standard errors of macronutrient intake by OJ consumption were calculated by the linearization (Taylor series) variance estimation method. The linear trends of macronutrient intakes and body parameters among OJ non-consumers and tertiles of OJ consumers were tested using linear contrasts after adjustment of age, gender, ethnicity and total energy intake. Where ANOVA results indicated significant difference, the Student $t$ test was conducted to compare macronutrient intakes between OJ non-consumers and consumers in each tertile of OJ consumption with Bonferroni correction, where statistical significance was defined as $P<0 \cdot 017$. Prevalence estimates were age-adjusted using the 2000 US Census ${ }^{(21)}$. In addition to being examined as categories, after testing if BMI (standardized as (raw value - mean)/SD) met the assumption of linearity in the logit, it was also evaluated as a continuous variable; odds ratios are presented for completeness. All $P$ values reported are two-tailed; statistical significance was defined as $P<0.05$ except for the $P$ values from Bonferroni correction.

\section{Results}

\section{Characteristics of orange juice consumers}

Sociodemographic, lifestyle and dietary characteristics of the 13971 participants by OJ consumption are described in Table 1 . In the present study, $25 \%$ of the participants consumed $\mathrm{OJ}$ as a beverage during at least one of the two non-consecutive recall days. Percentages of OJ consumption were different by gender, age, ethnicity, BMI and income level $(P<0 \cdot 05)$. Prevalence of OJ consumption was also different among those having different lifestyle or dietary behaviours including alcohol consumption, smoking, supplement use and regular exercise $(P<0 \cdot 05)$. Although OJ consumers overall drank more milk and less soft drinks than OJ non-consumers in our recent study ${ }^{(22)}$, in the present study OJ consumers in the highest tertile of OJ consumption $(\geq 222 \mathrm{ml} / \mathrm{d}$; $\geq 7.5 \mathrm{fl} \mathrm{oz} / \mathrm{d})$ had higher intakes of milk $(P<0.001)$ and soft drinks $(P<0.01)$ than OJ consumers in the lowest tertile $(<121 \mathrm{ml} / \mathrm{d}$; $<4 \cdot 1 \mathrm{fl} \mathrm{oz} / \mathrm{d}$ ).

\section{Macronutrient intakes by orange juice consumption}

Compared with participants who did not consume OJ or those in lower tertiles of OJ consumption, after adjusting for age, gender, ethnicity and energy intake, OJ consumers in the highest tertile group with $\geq 222 \mathrm{ml} / \mathrm{d}$ ( $\geq 7.5 \mathrm{fl} \mathrm{oz} / \mathrm{d}$ ) had higher intakes of total energy, carbohydrate, total sugar, total fat and fatty acids (SFA, MUFA and PUFA; $P<0 \cdot 01$; Tables 2 and 3). However, these linear trends for energy and macronutrient intakes remained unchanged in both children and adult groups when excluding OJ from the food list of items consumed $(P<0 \cdot 05)$. Percentages of energy from total fat were decreased by $\mathrm{OJ}$ consumption in both age groups $(P<0 \cdot 001)$. In addition, added sugar intake and percentage of energy from added sugar were not associated with OJ consumption in both age groups.

\section{Body composition by orange juice consumption}

Among children aged 4-18 years, after adjusted for age, gender, ethnicity and energy intake, OJ consumption had no association with BMI, WC, body fat $\%$ or odds ratio for overweight and obesity (Table 4). Taking a further look at age subgroups, children aged $4-8$ years did show increased odds ratio for obesity in the third tertile (data not shown), but age groups 9-13 years and 14-18 years had consistent results with the whole group of 4-18 years (data not shown). On the contrary, adults over 19 years who consumed more OJ had lower BMI, WC and body fat \% than non-consumers (Table 5). They also had lower odds ratio for overweight and obesity than non-consumers $(P<0 \cdot 01)$. In age subgroups, this negative association occurred mainly in the 31-50 years group (data not shown).

\section{Discussion}

The present study showed that OJ consumption had a negative association with BMI, WC, body fat $\%$ and odds ratio for overweight and obesity in adults. In children aged 4-18 years, OJ consumption had no association with BMI, WC, body fat \% or odds ratio for overweight and obesity. Nicklas et $a l^{(8)}$ reported similar data for $100 \%$ fruit juice in children 2-11 years of age. Although numerous previous studies have evaluated the association between growth parameters of children and 100\% fruit juice consumption, few of them evaluated the impact of specific types of fruit juices. When OJ is specifically 
Table 1 Sociodemographic, dietary and lifestyle characteristics according to OJ consumption among the US population aged $\geq 4$ years, NHANES 2003-2006*

\begin{tabular}{|c|c|c|c|c|c|c|c|c|c|c|}
\hline & \multirow[b]{2}{*}{$n$} & \multicolumn{2}{|c|}{$\begin{array}{l}\text { OJ non-consumers } \\
(\%)\end{array}$} & \multicolumn{2}{|c|}{$\begin{array}{c}\text { OJ consumers, } \mathrm{T} 1 \\
(\%)\end{array}$} & \multicolumn{2}{|c|}{$\begin{array}{c}\text { OJ consumers, T2 } \\
(\%)\end{array}$} & \multicolumn{2}{|c|}{$\begin{array}{c}\text { OJ consumers, T3 } \\
(\%)\end{array}$} & \multirow[b]{2}{*}{$P+$} \\
\hline & & Mean & SE & Mean & SE & Mean & SE & Mean & SE & \\
\hline \multicolumn{11}{|l|}{ OJ consumption range } \\
\hline $\mathrm{ml} / \mathrm{d}$ & & \multirow{2}{*}{\multicolumn{2}{|c|}{$\begin{array}{l}0 \\
0\end{array}$}} & \multirow{2}{*}{\multicolumn{2}{|c|}{$\begin{array}{l}<121 \\
<4 \cdot 1\end{array}$}} & \multirow{2}{*}{\multicolumn{2}{|c|}{$\begin{array}{l}121-222 \\
4 \cdot 1-7 \cdot 5\end{array}$}} & \multirow{2}{*}{\multicolumn{2}{|c|}{$\begin{array}{l}\geq 222 \\
\geq 7 \cdot 5\end{array}$}} & \\
\hline $\mathrm{fl} \mathrm{oz} / \mathrm{d}$ & & & & & & & & & & \\
\hline All & 13971 & $74 \cdot 8$ & $0 \cdot 6$ & $8 \cdot 3$ & $0 \cdot 4$ & $8 \cdot 3$ & $0 \cdot 4$ & $8 \cdot 6$ & $0 \cdot 4$ & \\
\hline \multicolumn{11}{|l|}{ Gender } \\
\hline Men & 6975 & $74 \cdot 2$ & $0 \cdot 7$ & $6 \cdot 6$ & $0 \cdot 3$ & $8 \cdot 1$ & 0.5 & $11 \cdot 0$ & 0.5 & $<0.001$ \\
\hline Women & 6996 & $75 \cdot 3$ & $0 \cdot 8$ & $9 \cdot 9$ & $0 \cdot 6$ & $8 \cdot 5$ & 0.5 & $6 \cdot 2$ & $0 \cdot 4$ & \\
\hline \multicolumn{11}{|l|}{ Age (years) } \\
\hline $4-8$ & 1534 & $69 \cdot 3$ & 1.5 & $17 \cdot 0$ & $1 \cdot 3$ & $9 \cdot 0$ & 0.9 & $4 \cdot 7$ & 0.8 & $<0.001$ \\
\hline $9-13$ & 1911 & 73.9 & $1 \cdot 4$ & $9 \cdot 7$ & 0.8 & $8 \cdot 7$ & $1 \cdot 0$ & $7 \cdot 7$ & 0.8 & \\
\hline $14-18$ & 2411 & $74 \cdot 5$ & $1 \cdot 4$ & $4 \cdot 8$ & $0 \cdot 7$ & $8 \cdot 4$ & 0.9 & $12 \cdot 3$ & $1 \cdot 0$ & \\
\hline $19-30$ & 1776 & $76 \cdot 9$ & $1 \cdot 2$ & $4 \cdot 9$ & 0.6 & $7 \cdot 3$ & 0.7 & $10 \cdot 9$ & 0.9 & \\
\hline $31-50$ & 2579 & $79 \cdot 6$ & $1 \cdot 1$ & $6 \cdot 0$ & 0.5 & $6 \cdot 3$ & 0.5 & $8 \cdot 1$ & 0.8 & \\
\hline$>50$ & 3760 & $70 \cdot 3$ & $1 \cdot 2$ & $11 \cdot 0$ & 0.6 & $10 \cdot 7$ & $0 \cdot 7$ & $8 \cdot 0$ & 0.5 & \\
\hline \multicolumn{11}{|l|}{ Ethnicity } \\
\hline Non-Hispanic whites & 5850 & $76 \cdot 3$ & $0 \cdot 8$ & $8 \cdot 0$ & $0 \cdot 4$ & $7 \cdot 8$ & 0.5 & $7 \cdot 9$ & $0 \cdot 4$ & $<0.01$ \\
\hline Non-Hispanic blacks & 3731 & $69 \cdot 3$ & $1 \cdot 0$ & $10 \cdot 0$ & $0 \cdot 6$ & $10 \cdot 5$ & 0.5 & $10 \cdot 2$ & 0.8 & \\
\hline Mexican Americans & 3453 & $72 \cdot 2$ & $1 \cdot 3$ & $8 \cdot 3$ & $0 \cdot 6$ & $9 \cdot 6$ & $0 \cdot 8$ & $10 \cdot 0$ & 0.9 & \\
\hline Others & $937 \cdot 0$ & $72 \cdot \overline{6}$ & 1.9 & $8 \cdot 3$ & $1 \cdot 3$ & $8 \cdot 2$ & $1 \cdot 1$ & $10 \cdot 9$ & $1 \cdot 1$ & \\
\hline \multicolumn{11}{|l|}{ BMI $\left(\mathrm{kg} / \mathrm{m}^{2}\right)$} \\
\hline$<18.5$ & 2442 & $72 \cdot 5$ & $1 \cdot 4$ & $13 \cdot 1$ & $1 \cdot 0$ & $8 \cdot 2$ & $0 \cdot 8$ & $6 \cdot 2$ & 0.9 & $<0.001$ \\
\hline $18 \cdot 5-24 \cdot 9$ & 4812 & 73.5 & 0.9 & $7 \cdot 2$ & 0.5 & $8 . \overline{9}$ & 0.6 & $10 \cdot 3$ & 0.6 & \\
\hline $25 \cdot 0-29 \cdot 9$ & 3450 & 73.9 & 0.8 & $8 \cdot 2$ & 0.5 & $8 \cdot 8$ & 0.5 & $9 \cdot 1$ & 0.6 & \\
\hline$\geq 30 \cdot 0$ & 3267 & $78 \cdot 3$ & $1 \cdot 3$ & $7 \cdot 5$ & 0.6 & $7 \cdot 2$ & $0 \cdot 8$ & $7 \cdot 0$ & 0.6 & \\
\hline \multicolumn{11}{|l|}{ PIR } \\
\hline$<1 \cdot 30$ & 5025 & $72 \cdot 9$ & $0 \cdot 8$ & $8 \cdot 4$ & 0.6 & $9 \cdot 3$ & 0.5 & $9 \cdot 4$ & 0.6 & $<0.05$ \\
\hline $1 \cdot 30-3 \cdot 49$ & 5185 & $75 \cdot 3$ & $1 \cdot 1$ & $9 \cdot 0$ & 0.5 & $7 \cdot 6$ & 0.7 & $8 \cdot 1$ & 0.5 & \\
\hline$\geq 3.50$ & 3761 & $75 \cdot 4$ & $1 \cdot 1$ & $7 \cdot 7$ & $0 \cdot 6$ & $8 \cdot 4$ & $0 \cdot 7$ & $8 \cdot 5$ & 0.6 & \\
\hline \multicolumn{11}{|l|}{ Beverage consumption } \\
\hline Other fruit juice $(\mathrm{g} / \mathrm{d})$ & 13971 & $29 \cdot 3$ & 1.5 & $32 \cdot 3$ & $2 \cdot 9$ & $28 \cdot 2$ & $3 \cdot 2$ & $37 \cdot 3$ & $6 \cdot 2$ & 0.33 \\
\hline Fruit drinks $(\mathrm{g} / \mathrm{d})$ & 13971 & $65 \cdot 5$ & $3 \cdot 2$ & $58 \cdot 1$ & $4 \cdot 1$ & $70 \cdot 4$ & $6 \cdot 9$ & $69 \cdot 2$ & $5 \cdot 8$ & 0.23 \\
\hline Milk $(g / d)$ & 13971 & 269 & $2 \cdot \overline{8}$ & 262 & 3.9 & 285 & $4 \cdot 6$ & 344 & $6 \cdot 2$ & $<0.001$ \\
\hline Soft drinks $(g / d)$ & 13971 & 435 & $15 \cdot 4$ & 277 & $24 \cdot 1$ & 313 & $16 \cdot 9$ & 348 & $16 \cdot 5$ & $<0.01$ \\
\hline \multicolumn{11}{|l|}{ Alcohol consumption§̧ } \\
\hline No & 2222 & $73 \cdot 9$ & $1 \cdot 2$ & $9 \cdot 5$ & $0 \cdot 8$ & $8 \cdot 6$ & 0.8 & $8 \cdot 0$ & $0 \cdot 6$ & $<0.05$ \\
\hline Yes & 5059 & $76 \cdot 5$ & 0.8 & $7 \cdot 0$ & 0.5 & $7 \cdot 9$ & 0.5 & 8.6 & 0.6 & \\
\hline \multicolumn{11}{|l|}{ Current smokingll } \\
\hline No & 2104 & $76 \cdot 1$ & $1 \cdot 2$ & $7 \cdot 3$ & 0.6 & $9 \cdot 6$ & $0 \cdot 8$ & $7 \cdot 0$ & 0.6 & $<0.001$ \\
\hline Yes & 1703 & $82 \cdot 8$ & $1 \cdot 1$ & $4 \cdot 7$ & $0 \cdot 7$ & $4 \cdot 8$ & 0.5 & $7 \cdot 8$ & $1 \cdot 0$ & \\
\hline Dietary supplement use & & & & & & & & & & \\
\hline No & 8514 & $76 \cdot 9$ & 0.9 & $7 \cdot 1$ & 0.5 & $7 \cdot 4$ & 0.4 & $8 \cdot 7$ & 0.4 & $<0.01$ \\
\hline Yes & 5444 & $72 \cdot 7$ & $0 \cdot 8$ & $9 \cdot 6$ & 0.5 & $9 \cdot 3$ & 0.5 & $8 \cdot 4$ & 0.5 & \\
\hline Exercise level $^{\star *}$ & & & & & & & & & & \\
\hline 0 & 2766 & $78 \cdot 3$ & $1 \cdot 2$ & $7 \cdot 0$ & 0.6 & $7 \cdot 3$ & 0.6 & $7 \cdot 5$ & 0.9 & $<0.01$ \\
\hline $\mathrm{T} 1$ & 2665 & $75 \cdot 5$ & $1 \cdot 1$ & 8.5 & 0.8 & $7 \cdot 7$ & 0.6 & 8.4 & 0.6 & \\
\hline T2 & 2631 & 73.5 & 0.8 & $6 \cdot 7$ & 0.7 & $9 \cdot 8$ & 0.8 & $10 \cdot 0$ & 0.6 & \\
\hline T3 & 2640 & $74 \cdot 1$ & $1 \cdot 2$ & $6 \cdot 8$ & 0.8 & 8.0 & 0.6 & $11 \cdot 1$ & $1 \cdot 0$ & \\
\hline
\end{tabular}

OJ, orange juice; NHANES, National Health and Nutrition Examination Survey; T1, T2 and T3, first, second and third tertiles; PIR, poverty income ratio. ${ }^{*} \mathrm{OJ}$ consumers were defined as those who reported consumption of non-sweetened $100 \%$ orange juice at least once in two non-consecutive $24 \mathrm{~h}$ diet recalls in NHANES 2003-2006.

$+P$ values were analysed by $\chi^{2}$ to test the differences in OJ consumption by gender, age subgroups, BMI, PIR, alcohol consumption, smoking, dietary supplement use and exercise level.

$\ddagger$ Ratio of the median family income over the poverty index. PIR $<1.3$ is required to be eligible for food assistance programmes.

\&Alcohol consumption: yes means having at least 12 alcoholic drinks/year.

॥Current smoking: yes means having smoked cigarettes, cigars or pipes or used chewing tobaccos or snuffs every day or some days.

-Dietary supplement use means taking any dietary supplements that include any vitamins, minerals or other dietary supplements at the time of interview.

${ }^{\star *}$ Exercise levels, expressed as MET (metabolic equivalent of task) scores, were calculated by combining the intensity level of the leisure-time activities reported with their mean duration and frequency.

reviewed, no positive association between OJ consumption and weight has been reported ${ }^{(4)}$.

To our knowledge, the present study is the first one that documents a negative association between OJ consumption and BMI along with other measures of body composition in adults. Prevalence of overweight and obesity was lower in OJ consumers than non-consumers in the general US population in this analysis. Of entire OJ consumers in the 
Table 2 Macronutrient intakes by OJ consumption in the US population aged 4-18 years, NHANES 2003-2006*, +

\begin{tabular}{|c|c|c|c|c|c|c|c|c|c|}
\hline \multirow[b]{2}{*}{ Nutrient } & \multicolumn{2}{|c|}{$\begin{array}{l}\text { OJ non-consumers } \\
\qquad(n 4040)\end{array}$} & \multicolumn{2}{|c|}{$\begin{array}{l}\text { OJ consumers, } \mathrm{T} 1 \\
(n \text { 605) }\end{array}$} & \multicolumn{2}{|c|}{$\begin{array}{l}\text { OJ consumers, T2 } \\
(n 606)\end{array}$} & \multicolumn{2}{|c|}{$\begin{array}{l}\text { OJ consumers, T3 } \\
(n \text { 605) }\end{array}$} & \multirow[b]{2}{*}{$P$ trend $\ddagger$} \\
\hline & Mean & SE & Mean & SE & Mean & SE & Mean & SE & \\
\hline \multicolumn{10}{|l|}{ OJ consumption range } \\
\hline $\mathrm{ml} / \mathrm{d}$ & \multirow{2}{*}{\multicolumn{2}{|c|}{$\begin{array}{l}0 \cdot 0 \\
0 \cdot 0\end{array}$}} & \multirow{2}{*}{\multicolumn{2}{|c|}{$<121$}} & \multicolumn{2}{|c|}{$121-216$} & \multirow{2}{*}{\multicolumn{2}{|c|}{$\geq 216$}} & \\
\hline $\mathrm{fl} \mathrm{oz} / \mathrm{d}$ & & & & $<4 \cdot 1$ & \multicolumn{2}{|c|}{$4 \cdot 1-7 \cdot 3$} & & $\geq 7 \cdot 3$ & \\
\hline \multicolumn{8}{|l|}{ Mean OJ consumption } & & \\
\hline $\mathrm{ml} / \mathrm{d}$ & \multirow{2}{*}{\multicolumn{2}{|c|}{$\begin{array}{l}0.0 \\
0.0\end{array}$}} & 83 & $0 \cdot 0$ & 160 & $0 \cdot 0$ & 370 & $5 \cdot 9$ & \\
\hline $\mathrm{fl} \mathrm{oz} / \mathrm{d}$ & & & $2 \cdot 8$ & 0.0 & $5 \cdot 4$ & 0.0 & $12 \cdot 5$ & $0 \cdot 2$ & \\
\hline \multicolumn{10}{|l|}{ Energy } \\
\hline $\mathrm{kJ} / \mathrm{d}$ & 8556 & 63 & 8251 & 151 & 8920 & 201 & 10209 & 285 & $<0.001$ \\
\hline $\mathrm{kcal} / \mathrm{d}$ & 2045 & 15 & 1972 & 36 & 2132 & 48 & 2440 & 68 & $<0.001$ \\
\hline$t$ Test $\S$ & \multicolumn{2}{|c|}{-} & \multicolumn{2}{|c|}{0.09} & \multicolumn{2}{|c|}{0.08} & \multirow{2}{*}{\multicolumn{2}{|c|}{$<0.017$}} & \\
\hline \multicolumn{8}{|l|}{ Energy excluding OJ } & & \\
\hline $\mathrm{kJ} / \mathrm{d}$ & 8556 & 63 & 8109 & 151 & 8632 & 201 & 9552 & 280 & $<0.05$ \\
\hline $\mathrm{kcal} / \mathrm{d}$ & 2045 & 15 & 1938 & 36 & 2063 & 48 & 2283 & 67 & $<0.05$ \\
\hline Protein $(g / d)$ & \multirow{2}{*}{\multicolumn{2}{|c|}{${ }^{72 \cdot 1}$}} & \multirow{2}{*}{\multicolumn{2}{|c|}{$\begin{array}{r}71 \cdot 4 \\
0.66\end{array}$}} & $74 \cdot 1$ & $1 \cdot 9$ & $87 \cdot 9$ & $2 \cdot 7$ & 0.67 \\
\hline$t$ Test & & & & & \multicolumn{2}{|c|}{0.28} & $<0$ & & \\
\hline Protein excluding OJ (g/d) & $72 \cdot 1$ & $0 \cdot 6$ & $70 \cdot 9$ & $1 \cdot 5$ & $73 \cdot 1$ & $1 \cdot 9$ & $85 \cdot 7$ & $2 \cdot 7$ & $0 \cdot 18$ \\
\hline $\begin{array}{l}\text { Carbohydrate }(\mathrm{g} / \mathrm{d}) \\
t \text { Test }\end{array}$ & & $2 \cdot 2$ & 262 & $5 \cdot 2$ & 286 & $6 \cdot 6$ & $\stackrel{333}{<0}$ & $8 \cdot 5$ & $<0.001$ \\
\hline Carbohydrates excluding OJ (g/d) & 272 & $2 \cdot 2$ & 254 & $5 \cdot 2$ & 270 & $6 \cdot 6$ & 296 & $8 \cdot 1$ & $<0.001$ \\
\hline Total sugar $(\mathrm{g} / \mathrm{d})$ & 136 & $1 \cdot 7$ & 129 & $2 \cdot 8$ & 144 & $3 \cdot 8$ & 171 & $4 \cdot 6$ & $<0.001$ \\
\hline$t$ Test & & & & & & & $<0$ & & \\
\hline Total sugar excluding OJ (g/d) & 120 & $1 \cdot 2$ & 105 & $2 \cdot 1$ & 115 & $2 \cdot 5$ & 129 & $2 \cdot 6$ & $<0.001$ \\
\hline Added sugar $(\mathrm{g} / \mathrm{d})$ & 78 & $1 \cdot 5$ & 73 & $3 \cdot 4$ & 80 & $3 \cdot 7$ & 80 & $3 \cdot 3$ & 0.31 \\
\hline Total fat $(\mathrm{g} / \mathrm{d})$ & $76 \cdot 6$ & 0.7 & $73 \cdot 4$ & $1 \cdot 8$ & $79 \cdot 0$ & $2 \cdot 3$ & $86 \cdot 4$ & 3.0 & $<0.001$ \\
\hline$t$ Test & & & & & & & $<0$. & & \\
\hline Total fat excluding OJ (g/d) & $81 \cdot 0$ & $0 \cdot 8$ & $74 \cdot 8$ & $1 \cdot 2$ & $77 \cdot 0$ & $1 \cdot 4$ & $89 \cdot 3$ & $1 \cdot 7$ & $<0.001$ \\
\hline Fatty acids & & & & & & & & & \\
\hline SFA $(g / d)$ & $27 \cdot 0$ & 0.3 & $25 \cdot 9$ & $0 \cdot 7$ & $27 \cdot 7$ & $0 \cdot 8$ & $30 \cdot 6$ & $1 \cdot 1$ & $<0.01$ \\
\hline $\operatorname{MUFA}(\mathrm{g} / \mathrm{d})$ & $28 \cdot 3$ & 0.3 & $27 \cdot 0$ & $0 \cdot 7$ & $29 \cdot 0$ & $0 \cdot 8$ & $31 \cdot 6$ & $1 \cdot 1$ & $<0.001$ \\
\hline PUFA $(g / d)$ & $15 \cdot 1$ & $15 \cdot 1$ & $14 \cdot 4$ & $0 \cdot 4$ & $15 \cdot 8$ & 0.7 & $16 \cdot 6$ & $0 \cdot 7$ & $<0.01$ \\
\hline Cholesterol (mg/d) & 225 & $2 \cdot 8$ & 245 & $8 \cdot 0$ & 231 & $5 \cdot 8$ & 290 & $11 \cdot 9$ & 0.44 \\
\hline$\% \mathrm{E}$ from protein/d & $14 \cdot 1$ & $0 \cdot 1$ & $14 \cdot 5$ & $0 \cdot 2$ & $13 \cdot 8$ & 0.2 & $14 \cdot 3$ & $0 \cdot 2$ & $0 \cdot 75$ \\
\hline$\%$ E from carbohydrate/d & $52 \cdot 9$ & $0 \cdot 2$ & $52 \cdot 8$ & 0.5 & $53 \cdot 8$ & $0 \cdot 4$ & $54 \cdot 6$ & $0 \cdot 4$ & $<0.001$ \\
\hline$\%$ E from added sugar/d & $16 \cdot 9$ & 0.3 & $16 \cdot 1$ & $0 \cdot 6$ & $16 \cdot 4$ & $0 \cdot 8$ & $14 \cdot 9$ & $0 \cdot 8$ & $0 \cdot 21$ \\
\hline$\%$ from fat/d & $33 \cdot 1$ & $0 \cdot 2$ & $32 \cdot 8$ & $0 \cdot 4$ & $32 \cdot 4$ & $0 \cdot 4$ & $31 \cdot 1$ & $0 \cdot 4$ & $<0.001$ \\
\hline
\end{tabular}

OJ, orange juice; NHANES, National Health and Nutrition Examination Survey; T1, T2 and T3, first, second and third tertiles; \%E, percentage of energy. *Estimation of nutrient intake was based on two non-consecutive $24 \mathrm{~h}$ diet recalls in NHANES $2003-2006$.

tOJ consumers were defined as those who reported consumption of non-sweetened $100 \%$ orange juice at least once in two non-consecutive $24 \mathrm{~h}$ diet recalls in NHANES 2003-2006.

$\ddagger P$ trend values were adjusted for age, gender, ethnicity and energy intake; $P$ value for energy intake was adjusted for age, gender and ethnicity. $P$ value was calculated across the mean of intake of $\mathrm{OJ}$ in each tertile using multivariate regression models.

$\S$ Student's $t$ tests were performed between OJ non-consumers and consumers in each tertile of OJ consumption with Bonferroni correction, where statistical significance was defined as $P<0.017$.

present study, 26\% were overweight and $20 \%$ were obese; while during the same period 2003-2006, 33\% of adults over 20 years in the USA were overweight and $33 \%$ were obese ${ }^{(23)}$. In addition, $32 \%$ of children and adolescents aged 2-19 years were overweight (BMI-for-age $>85$ th percentile) and $17 \%$ were obese (BMI-for-age $>$ 95th percentile) during 2007-2008 ${ }^{(24)}$.

For OJ consumers ingesting $<216 \mathrm{ml} / \mathrm{d}(<7 \cdot 3 \mathrm{fl} \mathrm{oz} / \mathrm{d})$, there was no association with energy intake in children. In the adult group, OJ consumers in the lowest tertile group ingesting $<121 \mathrm{ml} / \mathrm{d}(<4 \cdot 1 \mathrm{fl} \mathrm{oz})$, OJ consumption was inversely associated with energy intake. However, we found that energy intake was higher only in the group in the highest tertile of OJ consumption for both children and adults $(P<0 \cdot 017)$. This has been observed in previous studies ${ }^{(8,10)}$ and warrants further investigation to determine the source and effect of the additional energy.
However, it is premature to suggest that OJ solely contributes to this increase in energy, since the observed linear trends of increased energy intake remained after OJ was removed from the food list of items consumed. To determine the effect of this lower energy intake on weight status would require a sufficiently powered longitudinal study ${ }^{(8)}$. In addition, further investigation is warranted to understand potential mechanisms of OJ and weight control, since preliminary studies have documented the potential effectiveness of OJ consumption in weight control $^{(25,26)}$. For example, in an animal model, Titta et al. reported that blood orange juice inhibited fat accumulation in mice despite increased energy intake from sugar ${ }^{(25)}$.

In the present study, OJ consumption was positively correlated with milk consumption and negatively associated with carbonated soft drinks consumption, indicating that drinking OJ facilitates achieving nutrient adequacy and 
Table 3 Macronutrient intakes by OJ consumption in the US population aged $\geq 19$ years, NHANES 2003-2006*, +

\begin{tabular}{|c|c|c|c|c|c|c|c|c|c|}
\hline \multirow[b]{2}{*}{ Nutrient } & \multicolumn{2}{|c|}{$\begin{array}{l}\text { OJ non-consumers } \\
\qquad(n \text { 5921) }\end{array}$} & \multicolumn{2}{|c|}{$\begin{array}{l}\text { OJ consumers, } \mathrm{T} 1 \\
(n \text { 754) }\end{array}$} & \multicolumn{2}{|c|}{$\begin{array}{l}\text { OJ consumers, T2 } \\
(n 712)\end{array}$} & \multicolumn{2}{|c|}{$\begin{array}{l}\text { OJ consumers, T3 } \\
(n \text { 728) }\end{array}$} & \multirow[b]{2}{*}{$P$ trend $\ddagger$} \\
\hline & Mean & SE & Mean & SE & Mean & SE & Mean & SE & \\
\hline \multicolumn{10}{|l|}{ OJ consumption range } \\
\hline $\mathrm{ml} / \mathrm{d}$ & \multirow{2}{*}{\multicolumn{2}{|c|}{$\begin{array}{l}0.0 \\
0.0\end{array}$}} & \multirow{2}{*}{\multicolumn{2}{|c|}{$\begin{array}{l}<121 \\
<4 \cdot 1\end{array}$}} & \multirow{2}{*}{\multicolumn{2}{|c|}{$121-222$}} & \multicolumn{2}{|c|}{$\geq 222$} & \\
\hline $\mathrm{fl} \mathrm{oz} / \mathrm{d}$ & & & & & & $4 \cdot 1-7 \cdot 5$ & \multicolumn{2}{|c|}{$\geq 7 \cdot 5$} & \\
\hline \multicolumn{8}{|l|}{ Mean OJ consumption } & & \\
\hline $\mathrm{ml} / \mathrm{d}$ & \multirow{2}{*}{\multicolumn{2}{|c|}{$\begin{array}{l}0.0 \\
0.0\end{array}$}} & 89 & $0 \cdot 0$ & \multirow{2}{*}{$\begin{array}{l}169 \\
5 \cdot 7\end{array}$} & $0 \cdot 0$ & \multirow{2}{*}{$\begin{array}{l}363 \\
12 \cdot 3\end{array}$} & \multirow{2}{*}{$\begin{array}{l}6 \\
0.2\end{array}$} & \\
\hline $\mathrm{fl} \mathrm{oz/d}$ & & & $3 \cdot 0$ & 0.0 & & 0.0 & & & \\
\hline \multicolumn{10}{|l|}{ Energy } \\
\hline $\mathrm{kJ} / \mathrm{d}$ & 8941 & 71 & 8150 & 142 & 8862 & 176 & 10577 & 201 & $<0.001$ \\
\hline $\mathrm{kcal} / \mathrm{d}$ & 2137 & 17 & 1948 & 34 & 2118 & 42 & 2528 & 48 & $<0.001$ \\
\hline$t$ Test & \multirow{2}{*}{\multicolumn{2}{|c|}{-}} & \multicolumn{2}{|c|}{$<0.017$} & \multicolumn{2}{|c|}{0.68} & \multirow{2}{*}{\multicolumn{2}{|c|}{$<0.017$}} & \\
\hline \multicolumn{6}{|l|}{ Energy excluding OJ } & & & & \\
\hline $\mathrm{kJ} / \mathrm{d}$ & 8941 & 71 & 7991 & 142 & 8556 & 176 & 9920 & 201 & $<0.01$ \\
\hline $\mathrm{kcal} / \mathrm{d}$ & 2137 & 17 & 1910 & 34 & 2045 & 42 & 2371 & 48 & $<0.01$ \\
\hline Protein $(\mathrm{g} / \mathrm{d})$ & 83.5 & $0 \cdot 8$ & $76 \cdot 3$ & $1 \cdot 6$ & $80 \cdot 3$ & $1 \cdot 3$ & $95 \cdot 6$ & $2 \cdot 0$ & $0 \cdot 13$ \\
\hline$t$ Test & \multicolumn{2}{|c|}{-} & $<0$ & & & & $<0$ & & \\
\hline Protein excluding OJ (g/d) & $83 \cdot 5$ & $0 \cdot 8$ & $75 \cdot 7$ & $1 \cdot 6$ & $79 \cdot 3$ & $1 \cdot 3$ & $93 \cdot 4$ & $2 \cdot 0$ & $<0.01$ \\
\hline Carbohydrate $(\mathrm{g} / \mathrm{d})$ & 255 & $2 \cdot 0$ & 237 & $5 \cdot 0$ & 269 & $5 \cdot 7$ & 320 & $5 \cdot 7$ & $<0.001$ \\
\hline$t$ Test & & & $<0$ & & & & $<0$. & & \\
\hline Carbohydrates excluding OJ (g/d) & 255 & $2 \cdot 0$ & 228 & $5 \cdot 1$ & 252 & $5 \cdot 6$ & 283 & $5 \cdot 7$ & $<0.001$ \\
\hline Total sugar $(\mathrm{g} / \mathrm{d})$ & 115 & $1 \cdot 4$ & 106 & $3 \cdot 4$ & 127 & $3 \cdot 3$ & 159 & $3 \cdot 1$ & $<0.001$ \\
\hline$t$ Test & & & & & $<0$ & & $<0$. & & \\
\hline Total sugar excluding OJ (g/d) & 115 & $1 \cdot 4$ & 98 & 3.5 & 112 & $3 \cdot 3$ & 126 & $3 \cdot 1$ & $<0.01$ \\
\hline Added sugar $(\mathrm{g} / \mathrm{d})$ & 77 & $1 \cdot 0$ & 79 & $2 \cdot 6$ & 77 & $3 \cdot 1$ & 79 & $3 \cdot 7$ & $0 \cdot 75$ \\
\hline Total fat $(\mathrm{g} / \mathrm{d})$ & $82 \cdot 2$ & 0.9 & $74 \cdot 9$ & $1 \cdot 5$ & $77 \cdot 5$ & 1.9 & $90 \cdot 9$ & $2 \cdot 0$ & $<0.001$ \\
\hline$t$ Test & & & & & & & $<0$. & & \\
\hline Total fat excluding OJ (g/d) & $82 \cdot 2$ & 0.9 & $74 \cdot 8$ & 1.5 & $77 \cdot 3$ & 1.9 & $90 \cdot 5$ & $2 \cdot 0$ & $<0.001$ \\
\hline Fatty acids & & & & & & & & & \\
\hline SFA $(g / d)$ & $27 \cdot 4$ & 0.3 & $24 \cdot 6$ & 0.5 & $25 \cdot 3$ & $0 \cdot 7$ & $29 \cdot 6$ & $0 \cdot 7$ & $<0.001$ \\
\hline $\operatorname{MUFA}(g / d)$ & $30 \cdot 5$ & 0.4 & $27 \cdot 8$ & 0.6 & $28 \cdot 6$ & 0.8 & $33 \cdot 7$ & 0.7 & $<0.001$ \\
\hline PUFA ( $\mathrm{g} / \mathrm{d})$ & $17 \cdot 3$ & 0.2 & $16 \cdot 0$ & 0.4 & $16 \cdot 9$ & 0.5 & $19 \cdot 6$ & 0.5 & $<0.05$ \\
\hline Cholesterol (mg/d) & 289 & $3 \cdot 6$ & 285 & $10 \cdot 5$ & 267 & $5 \cdot 9$ & 336 & $9 \cdot 2$ & 0.08 \\
\hline$\%$ E from protein/d & $16 \cdot 3$ & $0 \cdot 1$ & $16 \cdot 0$ & 0.2 & $15 \cdot 5$ & 0.2 & $15 \cdot 6$ & 0.2 & $<0.01$ \\
\hline$\% \mathrm{E}$ from carbohydrate/d & $48 \cdot 8$ & $0 \cdot 2$ & $49 \cdot 7$ & 0.5 & $51 \cdot 9$ & $0 \cdot 3$ & $52 \cdot 1$ & $0 \cdot 3$ & $<0.001$ \\
\hline$\%$ E from added sugar/d & $17 \cdot 6$ & 0.3 & $18 \cdot 7$ & $0 \cdot 7$ & $17 \cdot 0$ & $0 \cdot 7$ & $14 \cdot 6$ & 0.7 & 0.91 \\
\hline$\%$ from fat/d & $34 \cdot 9$ & $0 \cdot 2$ & $34 \cdot 3$ & $0 \cdot 4$ & $32 \cdot 6$ & 0.3 & $32 \cdot 4$ & $0 \cdot 3$ & $<0.001$ \\
\hline
\end{tabular}

OJ, orange juice; NHANES, National Health and Nutrition Examination Survey; T1, T2 and T3, first, second and third tertiles; \%E, percentage of energy. ${ }^{*}$ Estimation of nutrient intake was based on two non-consecutive $24 \mathrm{~h}$ diet recalls in NHANES 2003-2006.

tOJ consumers were defined as those who reported consumption of non-sweetened $100 \%$ orange juice at least once in two non-consecutive $24 \mathrm{~h}$ diet recalls in NHANES 2003-2006.

$\ddagger P$ trend values were adjusted for age, gender, ethnicity and energy intake; $P$ value for energy intake was adjusted for age, gender and ethnicity. $P$ value was calculated across the mean of intake of $\mathrm{OJ}$ in each tertile using multivariate regression models.

$\S$ Student's $t$ tests were performed between OJ non-consumers and consumers in each tertile of OJ consumption with Bonferroni correction, where statistical significance was defined as $P<0.017$.

does not replace milk consumption. This finding is in good accordance with previous evidence suggesting that milk and fruit juice consumption was positively associated with the likelihood of achieving recommended intakes of vitamin $\mathrm{A}$, vitamin $\mathrm{B}_{12}$, folate, $\mathrm{Ca}, \mathrm{K}$ and $\mathrm{Mg}^{(27,28)}$. Harnack and colleagues ${ }^{(28)}$, in analysing data from the Continuing Survey of Food Intakes by Individuals (1994-1996), found that children who consumed more soda consumed less milk and fruit juice, and had lower mean intakes of nutrients related to milk and fruit juice. This inverse association between consumptions of fruit juice and soft drinks may be a potential pathway explaining why OJ consumption was not associated with risk of overweight or obesity among children and inversely associated in the adult group in our study. Future studies are needed to validate this hypothesis in different age groups. Also, future research should examine the extent to which
OJ consumption contributes to replacing soft drinks consumption and ameliorating or reducing risk of obesity although OJ consumption itself increases energy intake.

Our findings are interpreted based on several assumptions: first, the USDA food composition databases were constructed based on US representative food samples including varying cultivars, geographic origin, growing seasons, agricultural practices and analytical methods. Second, dietary intake data were based on two non-consecutive $24 \mathrm{~h}$ DR which might be a limitation of the current study since there is no scientific agreement on the minimum period of dietary data collection needed to obtain an approximation of usual intake or habitual dietary behaviours. However, the $24 \mathrm{~h}$ DR can produce adequate estimates of mean intake of a group that can be useful for contrasting the dietary status of the group with different levels of disease risks or health outcomes ${ }^{(29)}$. 
Table 4 Body composition by OJ consumption in the US population aged 4-18 years, NHANES 2003-2006*

\begin{tabular}{|c|c|c|c|c|c|c|c|c|c|}
\hline \multirow[b]{2}{*}{ Body measurement } & \multicolumn{2}{|c|}{ OJ non-consumers } & \multicolumn{2}{|c|}{ OJ consumers, $\mathrm{T} 1$} & \multicolumn{2}{|c|}{ OJ consumers, T2 } & \multicolumn{2}{|c|}{ OJ consumers, T3 } & \multirow[b]{2}{*}{$P$ trendt } \\
\hline & Mean & SE & Mean & SE & Mean & SE & Mean & SE & \\
\hline Weight-for-age Z-score $\neq$ & \multicolumn{2}{|c|}{$(n$ 4015) } & \multicolumn{2}{|c|}{$\begin{array}{c}0.47 \text { (n 649) }^{0.06} \\
\text { (n) }\end{array}$} & \multicolumn{2}{|c|}{${ }_{(n \text { 557) }}^{0.62}{ }^{0.08}$} & \multicolumn{2}{|c|}{${ }_{(n \text { 603) }}^{0.63}{ }^{0.08}$} & $0 \cdot 23$ \\
\hline BMI $\left(\mathrm{kg} / \mathrm{m}^{2}\right)$ & \multicolumn{2}{|c|}{${ }_{(n ~ 4015)}^{0 \cdot 1}$} & \multicolumn{2}{|c|}{$20 \cdot 7{ }_{(n \text { 649) }}^{0.2}$} & \multicolumn{2}{|c|}{$18 \cdot 8{ }_{(n \text { 557) }}^{0.2}$} & \multicolumn{2}{|c|}{$20 \cdot 6 \underbrace{0.3}_{(n 603)}$} & 0.28 \\
\hline WC $(\mathrm{cm})$ & \multicolumn{2}{|c|}{ 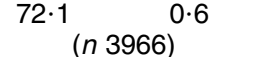 } & \multicolumn{2}{|c|}{${ }^{65 \cdot 4}(n 647)^{0.6}$} & \multicolumn{2}{|c|}{${ }^{71 \cdot 0}(n 552)^{0.7}$} & \multicolumn{2}{|c|}{$75 \cdot 6 \underset{(n 599)^{0.9}}{0.9}$} & 0.51 \\
\hline Triceps skinfold thickness (mm) & \multicolumn{2}{|c|}{$(n 3834)$} & \multicolumn{2}{|c|}{${ }_{(n \text { 633) }}^{13 \cdot 2}$} & \multicolumn{2}{|c|}{${ }_{(n \text { 537) }}^{14 \cdot 0}$} & \multicolumn{2}{|c|}{${ }^{14 \cdot 7}(n 580)^{0.4}$} & $1 \cdot 00$ \\
\hline \multirow[t]{2}{*}{ Body fat \%§ } & \multicolumn{2}{|c|}{$(n$ 1566) } & \multicolumn{2}{|c|}{$(n 212)$} & \multicolumn{2}{|c|}{$(n$ 212) } & \multicolumn{2}{|c|}{$(n 287)^{0.7}$} & 0.59 \\
\hline & OR & $95 \% \mathrm{Cl}$ & OR & $95 \% \mathrm{Cl}$ & OR & $95 \% \mathrm{Cl}$ & OR & $95 \% \mathrm{Cl}$ & \\
\hline \multirow{2}{*}{$\begin{array}{l}\text { BMI-for-age percentile } \geq 85 \text { th } \\
\text { BMI-for-age percentile } \geq 95 \text { th }\end{array}$} & \multicolumn{2}{|c|}{$\begin{array}{c}1 \cdot 00 \quad \text { Ref. } \\
(n \text { 4009) }\end{array}$} & \multicolumn{2}{|c|}{$\begin{array}{c}0.86 \quad 0.69,1.08 \\
(n \text { 649) }\end{array}$} & \multicolumn{2}{|c|}{$\begin{array}{c}1.06 \quad 0.78,1.45 \\
(n 557)\end{array}$} & \multicolumn{2}{|c|}{$\begin{array}{c}1.09 \quad 0.84,1.43 \\
(n \text { 603) }\end{array}$} & $0 \cdot 24$ \\
\hline & $\begin{array}{r}1 \cdot 00 \\
(n\end{array}$ & Ref. & $0 \cdot 80$ & $\begin{array}{l}0.58,1 \cdot 10 \\
49)\end{array}$ & $\begin{array}{r}1.06 \\
(n\end{array}$ & $\begin{array}{l}0.77,1 \cdot 45 \\
57)\end{array}$ & $\begin{array}{r}1 \cdot 02 \\
(n\end{array}$ & $\begin{array}{l}0 \cdot 70,1 \cdot 49 \\
03)\end{array}$ & 0.53 \\
\hline
\end{tabular}

OJ, orange juice; NHANES, National Health and Nutrition Examination Survey; T1, T2 and T3, first, second and third tertiles; WC, waist circumference; body fat $\%$, percentage of body fat; Ref., referent category.

${ }^{*} \mathrm{OJ}$ consumers were defined as those who reported consumption of non-sweetened $100 \%$ orange juice at least once in two non-consecutive $24 \mathrm{~h}$ diet recalls in NHANES 2003-2006.

tAdjusted for age, gender, ethnicity and energy intake.

$\ddagger$ Weight-for-age Z-score was calculated using 2000 Centers for Disease Control and Prevention growth chart ${ }^{(20)}$

§Body fat \% data are based on NHANES 2003-2004 (aged $\geq 8$ years) and calculated by dividing fat mass by total mass.

Table 5 Body composition by OJ consumption in the US population aged $\geq 19$ years, NHANES 2003-2006*

\begin{tabular}{|c|c|c|c|c|c|c|c|c|c|}
\hline \multirow[b]{2}{*}{ Body measurement } & \multicolumn{2}{|c|}{ OJ non-consumers } & \multicolumn{2}{|c|}{ OJ consumers, $\mathrm{T} 1$} & \multicolumn{2}{|c|}{ OJ consumers, T2 } & \multicolumn{2}{|c|}{ OJ consumers, T3 } & \multirow[b]{2}{*}{$P$ trendt } \\
\hline & Mean & SE & Mean & SE & Mean & SE & Mean & SE & \\
\hline Weight (kg) & \multicolumn{2}{|c|}{$(n 5845)$} & \multicolumn{2}{|c|}{$(n 744)$} & \multicolumn{2}{|c|}{${ }_{(n \text { 696) }}^{78.6}$} & \multicolumn{2}{|c|}{${ }_{(n 719)}^{81 \cdot 7}$} & $<0.01$ \\
\hline BMI $\left(\mathrm{kg} / \mathrm{m}^{2}\right)$ & \multicolumn{2}{|c|}{$(n 5845)$} & \multicolumn{2}{|c|}{$(n$ 744) } & \multicolumn{2}{|c|}{$(n 696)$} & \multicolumn{2}{|c|}{$(n 719)$} & $<0.001$ \\
\hline WC (cm) & \multicolumn{2}{|c|}{${ }_{(n 5718)^{08.1}}^{0.4}$} & $96 \cdot 8$ & 17) $0 \cdot 7$ & $95 \cdot 4$ & (71) $0 \cdot 7$ & $96 \cdot 1$ & $01)^{0 \cdot 8}$ & $<0.001$ \\
\hline Triceps skinfold thickness $(\mathrm{mm})$ & \multicolumn{2}{|c|}{$(n$ 5172) } & \multicolumn{2}{|c|}{$(n 660)$} & \multicolumn{2}{|c|}{$(n 617)$} & \multicolumn{2}{|c|}{$(n 650)$} & 0.05 \\
\hline \multirow[t]{2}{*}{ Body fat \%¥ } & \multicolumn{2}{|c|}{ (n 2925) } & $36 \cdot 0$ & 409) & $34 \cdot 1$ & $366)^{0.6}$ & \multicolumn{2}{|c|}{$(n$ 362) } & $<0.001$ \\
\hline & OR & $95 \% \mathrm{Cl}$ & OR & $95 \% \mathrm{Cl}$ & OR & $95 \% \mathrm{Cl}$ & OR & $95 \% \mathrm{Cl}$ & \\
\hline $\mathrm{BMI} \geq 25 \cdot 0 \mathrm{~kg} / \mathrm{m}^{2}$ & \multicolumn{2}{|c|}{$\begin{array}{c}1 \cdot 00 \quad \text { Ref. } \\
(n \text { 2925) }\end{array}$} & \multicolumn{2}{|c|}{$\begin{array}{c}1 \cdot 02 \underset{(n)}{0} 0 \cdot 86,1 \cdot 22 \\
(n) 9)\end{array}$} & \multicolumn{2}{|c|}{$\begin{array}{c}0.84 \underset{(n) 366)}{0.65,1 \cdot 08} \\
\text { (n }\end{array}$} & \multicolumn{2}{|c|}{$\begin{array}{c}0.69 \quad 0.56,0.84 \\
(n 362)\end{array}$} & $<0.001$ \\
\hline $\mathrm{BMI} \geq 30 \cdot 0 \mathrm{~kg} / \mathrm{m}^{2}$ & \multicolumn{2}{|c|}{$(n$ 2925) } & \multicolumn{2}{|c|}{$(n$ 409) } & $0 \cdot 73$ & $\begin{array}{l}0.56,0.96 \\
366)\end{array}$ & 0.68 & $\begin{array}{l}0.53,0.87 \\
62)\end{array}$ & $<0.01$ \\
\hline
\end{tabular}

OJ, orange juice; NHANES, National Health and Nutrition Examination Survey; T1, T2 and T3, first, second and third tertiles; WC, waist circumference; body fat $\%$, percentage of body fat; Ref., referent category.

${ }^{*} \mathrm{OJ}$ consumers were defined as those who reported consumption of non-sweetened $100 \%$ orange juice at least once in two non-consecutive $24 \mathrm{~h}$ diet recalls in NHANES 2003-2006.

tAdjusted for age, gender, ethnicity and energy intake.

¥Body fat $\%$ data are based on NHANES 2003-2004 (aged $\geq 8$ years) and calculated by dividing fat mass by total mass.

Last, the present study was based on cross-sectional data and we cannot draw any cause-and-effect conclusion on the impact of OJ consumption on weight status.

\section{Conclusions}

OJ consumption was associated with healthier body composition (lower BMI, WC and body fat \%) in adults, and there were no significant associations between
OJ consumption and body composition in children and adolescents. OJ consumption had no association with BMI, WC, body fat $\%$ or odds ratio for overweight and obesity. When we took a further look at age subgroups, children aged $4-8$ years did show increased odds ratio for obesity in the third tertile, but other age subgroups had consistent results with the whole group of 4-18 years. The encouraging results from our study, however, suggest that larger, longitudinal studies are warranted. 


\section{Acknowledgements}

Parts of the current paper were presented at the 2011 Experimental Biology Meeting, Washington, DC, in April 2011. The research was funded by PepsiCo Inc. B.L. is an employee of PepsiCo Inc. and possibly has a financial interest in the outcome of the study. The other authors bear no conflict of interest regarding the present manuscript. O.K.C. designed the study. B.L. participated in the study development. S.-J.C. was the statistician and provided technical support and advice as a member of the project's steering group. Y.W. analysed and interpreted the data and prepared the manuscript. M.Y., C.G.D., S-G.L. and W.L. helped analyse the data and review the paper. All authors reviewed the final version of the manuscript, approved it for publication and take public responsibility for its content.

\section{References}

1. Dennison BA, Rockwell HL \& Baker SL (1997) Excess fruit juice consumption by preschool-aged children is associated with short stature and obesity. Pediatrics 99, $15-22$.

2. Committee on Nutrition (2001) American Academy of Pediatrics: The use and misuse of fruit juice in pediatrics. Pediatrics 107, 1210-1213.

3. Dennison BA (1996) Fruit juice consumption by infants and children: a review. J Am Coll Nutr 15, 5 Suppl., 4S-11S.

4. Dennison BA, Rockwell HL, Nichols MJ et al. (1999) Children's growth parameters vary by type of fruit juice consumed. J Am Coll Nutr 18, 346-352.

5. Tanasescu M, Ferris AM, Himmelgreen DA et al. (2000) Biobehavioral factors are associated with obesity in Puerto Rican children. J Nutr 130, 1734-1742.

6. Skinner JD, Carruth BR, Moran J 3rd et al. (1999) Fruit juice intake is not related to children's growth. Pediatrics 103, 58-64.

7. Newby PK, Peterson KE, Berkey CS et al. (2004) Beverage consumption is not associated with changes in weight and body mass index among low-income preschool children in North Dakota. J Am Diet Assoc 104, 1086-1094.

8. Nicklas TA, O'Neil CE \& Kleinman R (2008) Association between $100 \%$ juice consumption and nutrient intake and weight of children aged 2 to 11 years. Arch Pediatr Adolesc Med 162, 557-565.

9. LaRowe TL, Moeller SM \& Adams A (2007) Beverage patterns, diet quality, and body mass index of US preschool and school-aged children. J Am Diet Assoc 107, 1124-1133.

10. O'Connor TM, Yang SJ \& Nicklas TA (2006) Beverage intake among preschool children and its effect on weight status. Pediatrics 118, e1010-e1018.

11. Rampersaud GC (2007) A comparison of nutrient density scores for 100\% fruit juices. J Food Sci 72, issue 4, S261-S266.
12. Franke AA, Cooney RV, Henning SM et al. (2005) Bioavailability and antioxidant effects of orange juice components in humans. J Agric Food Chem 53, 5170-5178.

13. National Center for Health Statistics (2005) Analytical and Reporting Guidelines: The National Health and Nutrition Examination Survey (NHANES). Hyattsville, MD: Centers for Disease Control and Prevention.

14. National Center for Health Statistics (2006) National Health and Nutrition Examination Survey, 2003-2004 Data Files. Hyattsville, MD: Centers for Disease Control and Prevention.

15. National Center for Health Statistics (2008) National Health and Nutrition Examination Survey, 2005-2006 Data Files. Hyattsville, MD: Centers for Disease Control and Prevention.

16. US Department of Agriculture, Agricultural Research Service (2010) USDA Automated Multiple-Pass Method. http://www.ars.usda.gov/Services/docs.htm?docid $=7710$ (accessed October 2010).

17. US Department of Agriculture, Agricultural Research Service (2006) USDA Database for the Added Sugars Content of Selected Foods, Release 1. Beltsville, MD: USDA.

18. National Heart, Lung, and Blood Institute (2000) Practice Guide to the Evaluation and Treatment of Overweight and Obesity in Adults. Bethesda, MD: US Department of Health and Human Services.

19. Krebs NF, Himes JH, Jacobson D et al. (2007) Assessment of child and adolescent overweight and obesity. Pediatrics 120, Suppl. 4, 193S-228S.

20. Kuczmarski RJ, Ogden CL, Grummer-Strawn LM et al. (2000) CDC growth charts: United States. Adv Data issue 314, 1-27.

21. Klein RJ \& Schoenborn C (2001) Age Adjustment Using the 2000 Projected US Population. Healthy People Statistical Notes no. 20. Hyattsville, MD: National Center for Health Statistics.

22. Chun OK, Yang M, Wang Y et al. (2011) Contribution of orange juice consumption to micronutrient adequacy in US population. Presented at Experimental Biology Meeting, Washington, DC, 9-13 April 2011.

23. Centers for Disease Control and Prevention (2008) Prevalence of overweight, obesity and extreme obesity among adults: United States, trends 1976-80 through 2005-2006. http://www.cdc.gov/nchs/data/hestat/overweight/overweight_ adult.pdf (accessed October 2010).

24. Ogden CL, Carroll MD, Curtin LR et al. (2010) Prevalence of high body mass index in US children and adolescents, 2007-2008. JAMA 303, 275-276.

25. Titta L, Trinei M, Stendardo M et al. (2009) Blood orange juice inhibits fat accumulation in mice. Int J Obes (Lond) 34, 578-588.

26. Ghanim H, Sia CL, Upadhyay M et al. (2010) Orange juice neutralizes the proinflammatory effect of a high-fat, highcarbohydrate meal and prevents endotoxin increase and Toll-like receptor expression. Am J Clin Nutr 91, 940-949.

27. Ballew C, Kuester S \& Gillespie C (2000) Beverage choices affect adequacy of children's nutrient intakes. Arch Pediatr Adolesc Med 154, 1148-1152.

28. Harnack L, Stang J \& Story M (1999) Soft drink consumption among US children and adolescents: nutritional consequences. J Am Diet Assoc 99, 436-441.

29. Byers T (1992) Nutrition monitoring and surveillance. In Nutritional Epidemiology, 2nd ed. pp. 347-356 [W Willett, editor]. New York: Oxford University Press. 